Nachrichten der Gesellschaft für Kinder- und Jugendrheumatologie

\title{
Die Kommissionen/Arbeitskreise der GKJR stellen sich vor
}

Nach Vorstellung der Kommission Bewegung und Sport in Heft 1/2021 der a $+r$ folgt nun der Bericht der Kommission Pharmakotherapie und Leitlinien. Da im letzten Jahr die gemeinsame Jahrestagung mit der DGKJ nur virtuell stattfinden konnte und damit verbunden die Mitgliederversammlung der GKJR ausfallen musste, hatte der Beirat im Rahmen des Strategiemeetings im September beschlossen, die Arbeit der Kommissionen im Rahmen kurzer Beiträge darzustellen.

\section{Kommission Pharmako- therapie und Leitlinien}

Es ist uns eine Freude, kurz zu bündeln, was sich seit der letzten Präsenztagung in Dresden 2019 in unserer Kommission ereignet hat.

\section{Leitlinien}

Im Dezember 2019 konnte nach mehrjähriger Vorarbeit die Leitlinie „Therapie der juvenilen idiopathischen Arthritis“ (027-020) auf der AWMF-Seite publiziert und zugänglich gemacht werden. In 2 deutschsprachigen Zeitschriften wurden die Ergebnisse seither publiziert (arthritis + rheuma 2020; 40: 260-269, Monatsschr Kinderheilkd 2020; 168: 947-949). Zahlreiche Mitglieder der Kommission und der Fachgesellschaft sind mit Aktualisierungen bestehender Leitlinien, z. T. unter Federführung, z. T. unter Beteiligung der GKJR befasst. Unter Federführung der GKJR wurde ganz aktuell die Leitlinie zum Kawasaki-Syndrom (185003) abgeschlossen und ist seither auf der AWMF-Seite zugänglich. Darüber hinaus wurden im Jahr 2020 unter Beteiligung engagierter GKJR-Mitglieder folgende Leitlinien aktualisiert (in Klammern finden sich jeweils die federführenden Fachgesellschaften):

\footnotetext{
- Muskuloskelettale Schmerzen bei Kindern und Jugendlichen (DGKJ), - Lymphknotenvergrößerung (GPOH),
}

- Diagnostik und Therapie des kutanen Lupus erythematodes (DDG).

Zu den angemeldeten Leitlinienvorhaben, bei denen die GKJR beteiligt ist, gehört u. a. die Leitlinie zu Inflammatorischen Erkrankungen des Kiefergelenks. In Vorbereitung ist des Weiteren eine interdisziplinäre Leitlinie für den cSLE/jSLE.

\section{Aufklärungsbögen zu krankheitsmodifizierenden Medikamenten (DMARDs)}

Schon lange bestand der Wunsch, die im Alltag der Praxis oder Spezialambulanz doch sehr handlichen Informationsbögen für Familien über DMARDs zu überarbeiten. Nicht nur waren Teile überholt (z. B. ab welchem Alter ein bestimmtes Biologikum zugelassen ist), sondern es sollten Informationen zu Themen wie z. B. Empfehlung zu Impfungen oder leicht erhöhtem Entartungsrisiko bei Immunsuppressiva/Biologika vereinheitlicht werden.

Neue Informationsbögen wurden für folgende Medikamente erstellt: Pamidronat, Dapson, Canakinumab, Secukinumab und Belimumab. Überarbeitet wurden alle bisher auf der Homepage der GKJR vorhandenen Medikamenteninformationsbögen. Im Einzelnen sind das alphabetisch geordnet: Anakinra, Azathioprin, Colchizin, Cyclosporin, Cyclophosphamid, HCQ, Kortikosteroide, Leflunomid, MMF, MTX, NSAR, Rituximab, anti-TNFs (Adalimumab, Certolizumab, Etanercept, Golimumab) und Tocilizumab.

Hier ist auch der Moment, die freiwilligen, fleißigen und geduldigen Mitstreiterinnen und Mitstreiter namentlich zu erwähnen: Andreas Urban (Amberg), Fabian Speth (Hamburg seit 12/2020, zuvor Rostock), Thomas Keller (Augsburg), Reiner Berendes (Landshut), Frank Dressler (Hannover), Frank Weller (Bremen), Sonja Mrusek (Ba-
den-Baden), Ivan Foeldvari (Hamburg), Elisabeth Weissbarth-Riedel (Hamburg).

Ich hoffe, ich habe niemanden vergessen.

\section{Handlungsempfehlungen der Gesellschaft für Kinder- und Jugendrheumatologie bzgl. SARS-CoV-2}

Zum Thema Immunsuppressiva und COVIDRisiko bzw. positivem SARS-CoV-2-Abstrich bei Kindern mit rheumatischen Erkrankungen unter Therapie mit DMARDs hat die GKJR gemeinsam mit Vertretern der DGPI eine Stellungnahme zu Handlungsempfehlungen bei Kindern und Jugendlichen mit rheumatischen oder autoinflammatorischen Erkrankungen (Version 2.0) erarbeitet. Diese ist zu finden unter: www.gkjr.de/ wp-content/uploads/2020/03/infektionmit-coronavirus-sars-cov-2_v2.pdf

\section{Positive Nutzenbewertung von Belimumab für den pädiatrischen/ juvenilen SLE}

Hier war die Fachgesellschaft (u. a. vom Hersteller GlaxoSmithKline GmbH \& Co. KG) gebeten worden, sich zu positionieren. Das IQWiG war zum Schluss gekommen, dass Belimumab im Kindes- und Jugendalter keinen Mehrwert gegenüber der Standardtherapie habe. Unter Federführung von Klaus Tenbrock, Kirsten Minden und Gerd Horneff hatten wir eine Stellungnahme verfasst und darauf hingewiesen, dass u. a. aufgrund von Schwächen der angewandten Scores zur Messung der Krankheitsaktivität die Nutzenbewertung für das Kindes- und Jugendalter in der PLUTO-Studie zu negativ evaluiert wurde. Gerd Horneff und Kirsten Minden hatten diese Einwände bei der Anhörung im Mai 2020 in Berlin vorgetragen.

Fazit: „Der G-BA betont in seinen tragenden Gründen, wie entscheidend sowohl das Stellungnahmeverfahren als auch die mündliche Anhörung waren, da sie die grundsätzliche 
Eignung der PLUTO-Studie für die Nutzenbewertung bei jSLE-Patienten mit hoher Krankheitsaktivität deutlich gemacht haben.“ (Zitat von Heike Carnarius, GSK)

Die Treffen der Kommission finden mindestens einmal im Jahr im Rahmen der Jahrestagungen der Gesellschaft statt. Dies war erwartungsgemäß 2020 durch Reiseeinschränkungen nicht möglich. Hingegen fanden zahlreiche Telefonkonferenzen zwischen den Sprechern, v. a. aber mit Mitstreitern aus der Kommission und aus der GKJR statt. Themen waren außer den genannten
Leitlinien auch die sich über 2 Jahre streckende Überarbeitung bzw. Neuerstellung der Medikamentenbögen. Wir danken hier noch einmal den zahlreichen Beitragenden aus der Kommission.

Die Kontaktaufnahme kann über einen der Kommissionssprecher erfolgen.

Prasad Oommen, Universitätsklinikum Düsseldorf (prasad.oommen@med.uniduesseldorf.de)

Catharina Schütz, Universitätsklinikum Dresden (catharina.schuetz@ukdd.de)

\section{KONTAKTADRESSE}

Gesellschaft für Kinder- und Jugendrheumatologie

Geschäftsstelle

c/o Deutsches Rheuma-Forschungszentrum (DRFZ)

Programmbereich Epidemiologie Gabriele Berg

Charitéplatz 1, 10117 Berlin

Tel.: 030/28 460-632

Fax: 030/28 460-744

E-Mail: gabriele.berg@drfz.de

IMPRESSUM

Verantwortlich für den Inhalt

Martina Niewerth

GKJR-Geschäftsstelle, Deutsches

Rheuma- Forschungszentrum, Berlin 